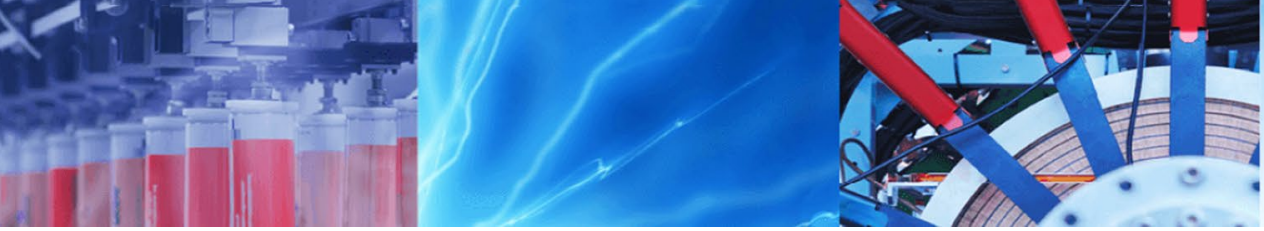

Research Article

\title{
Fabrication and property evaluation of Al $6061+x \%$ (RHA + TiC) hybrid metal matrix composite
}

\author{
A. Chinnamahammad Bhasha ${ }^{1} \cdot$ K. Balamurugan ${ }^{2}$
}

(c) Springer Nature Switzerland AG 2019

\begin{abstract}
The present work aims in understanding the mechanical properties and strengthening behavior of Al 6061 based particulate hybrid aluminium matrix composites (PHAMCs) reinforced with rice husk ash (RHA) and TiC particles (sizes; $10 \mu \mathrm{m}$ ) fabricated by liquid metallurgical route. RHA + TiC particles ( $3 \mathrm{wt} \%, 5 \mathrm{wt} \%, 7 \mathrm{wt} \%$, and $9 \mathrm{wt} \%$ ) are added in equal proposition in the hybrid composite which is fabricated through stir-casting technique. Samples are named as S1, S2, S3, S4 based on the proposition of RHA and TiC, whereas Al 6061 alloy is casted and named as S0. Fabricated composites were homogenized at $400^{\circ} \mathrm{C}$ for $1 \mathrm{~h}$ and then aged to $115^{\circ} \mathrm{C}$ for $24 \mathrm{~h}$. X-ray diffraction result confirms the presence of principle elements like Al, TiC, Si and oxygen without the formation of any intermetallic elements. Property of PHAMCs such as ultimate tensile strength, yield strength, and micro Vickers hardness had improved by $31 \%, 17.1 \%$, and $18 \%$, respectively with increase in the percentage of reinforcement. Addition of reinforcement slightly lowers the mechanical properties of PHAMCs which is due to agglomerations and porosity. Reinforcement with more than 7\% of RHA and TiC in matrix significantly effect the density, impact energy and percentage of elongation to a maximum of $3.88 \%, 28.52 \%$, and $29.91 \%$, respectively. Microstructure of the fractured surfaces are analysed through SEM and it is reported.
\end{abstract}

Keywords PHAMCS · Al $6061 \cdot$ Stir-casting $\cdot$ SEM $\cdot$ XRD

\section{Introduction}

Conventional metallic alloys are being replaced with metal matrix composites (MMC) in many engineering applications such as defence, marine, sports and recreation industries, etc. In-particular, MMC has almost replaced all the conventional materials in aerospace and automobile applications [1]. MMC possess the advantages of different properties of metallic, ceramic and polymer materials to form a mixture with superior properties [2]. Among the light metals, Al and $\mathrm{Mg}$ are used as a base matrix and reinforced with foreign materials to enhance the material properties. Aluminum matrix composite has gain importance than $\mathrm{Mg}$ because of ease of fabrication [3]. AA6061 series possess moderate strength with lightweight and it is widely used for structural, aviation and automobile applications [4].
Various ceramic particles like carbides, nitrides, and oxides are reinforced with aluminium matrix to form PHAMCs. PHAMCs perform excellent metallic properties than MMC [5]. Reinforcement of ceramic particles in Al 6061 matrix will increase the density of the composite. Selection of suitable reinforcement while in fabrication of composite will yield to have low density PHAMCs. Fabrication of PHAMCs with lightweight, more strength, stiffness, better wear, and corrosion resistance for the structural applications is challenging task [6]. Industrial by-products or Agro-waste derivates as a reinforcement material in the aluminum matrix has drawn a lot of interest in recent years among researchers [7].

Liquid metallurgical route (casting) and solid-state route (powder metallurgy) are the general methods used to fabricate PHAMCs, among them, stir casting process is a

K. Balamurugan, kbalan2000@gmail.com | ${ }^{1}$ Research Scholar, Department of Mechanical Engineering, VFSTR (Deemed to be University), Guntur, AP 522213, India. ${ }^{2}$ Department of Mechanical Engineering, VFSTR (Deemed to be University), Guntur, AP 522213, India.

SN Applied Sciences (2019) 1:977 | https://doi.org/10.1007/s42452-019-1016-0 
suitable and cost-effective technique. Mechanical properties of AA6061 matrix reinforced with $\mathrm{Al}_{2} \mathrm{O}_{3} / \mathrm{SiC}$ fabricated through stir casting has shown better results than powder metallurgy method [8]. Limited studies are available on AA matrix composite reinforced with agro-waste. Use of RHA as reinforcement in the matrix will create some serious issue such as agglomeration that significantly reduces the mechanical properties of the composites [9].

In Al 1100 series coconut shell ash is used as reinforcement with varied proportion and found that the specific strength and wear resistances of the composites are increased by $70 \%$ and $15 \%$, respectively [10]. Increase of RHA composition in AA7075/RHA/B ${ }_{4} \mathrm{C}$ mixture will produce low density composite with increased weight fraction [11]. Density, hardness, metallic properties of the composite improves with reduction in percentage of elongation and impact strength on addition of TiC as reinforcement in AA6063 composite [12]. RHA in Al/SiC/RHA hybrid composite will lead to high porosity that substantially effects the surface profile of the machined composite [13]. Addition of RHA as reinforcement in A356.2 matrix improves the hardness and density of the composite [14]. Abundant quantity of silica can be obtained through agro waste with low cost and can be used as the reinforcement material [15]. The presence of $\mathrm{SiO}_{2}, \mathrm{Al}_{2} \mathrm{O}_{3}, \mathrm{MgO}, \mathrm{CaO}$ and other refractory particles in RHA helps in improving the physical, mechanical and tribological properties [16].

The influence of RHA in the matrix predominantly determine the properties of the composite attracted the researchers to a greater extent. Some studies have reported that using RHA as the reinforcement in the Aluminium matrix. $\mathrm{TiC}$ is used as secondary reinforcement because for its unique properties such as wear resistance, superior hardness, stability, stable at high temperatures. In the present work, PHAMCs are prepared with different equal weight fractions of RHA and TiC particulates (i.e. $3 \%+3 \%, 5 \%+5 \%, 7 \%+7 \%$, and $9 \%+9 \%$ ) through double stir casting process technique. The influence of reinforcement particles on tensile test, impact test, and micro
Vickers hardness test of PHAMC's in tempered conditions have been studied and the microstructural characterization on the fractured surface is performed through SEM and XRD analysis.

\section{Materials and methods}

\subsection{Materials}

Al 6061 alloy is purchased in the form of ingots from Renuka Engineering Pvt. Ltd., Hyderabad and it is used as the base material. TiC and RHA are used as the reinforcement particulates. Chemically pure TiC particles of $10 \mu \mathrm{m}$ are procured from Renuka engineering Pvt. Ltd., Hyderabad. The rice husk is collected from Guntur region, Andhra Pradesh, India. Rice husk is washed with water to remove the dust particles and clay if any and dried at room temperature for 1 day. To remove the moisture and organic matter, rice husk is placed inside muffle furnace for $1 \mathrm{~h}$ at a temperature of $210^{\circ} \mathrm{C}$. During this operation, the color of husk changed from yellowish to black because of the charring of organic matter. RHA particles are then sieved through $100 \mu \mathrm{m}$ sieve and then heated to a temperature of $650{ }^{\circ} \mathrm{C}$ for $24 \mathrm{~h}$ to remove any carbonaceous matter. Among the projected techniques, Prasad et al. [17] has found an easy way for extraction of silica from RHA. At this stage ash changes from black to grayish white color. Subsequently, the collected ash is ball milled for $16 \mathrm{~h}$ to get the particle size of $10 \mu \mathrm{m}$. The different stages of rice husk to RHA are shown in Fig. 1a-d. The particle sizes of $\mathrm{TiC}$ and $\mathrm{RHA}$ is in the range of $10-12 \mu \mathrm{m}$.

\subsection{Methods}

Double stir casting process is employed to fabricate PHAMCs. The experimental arrangements and the casted samples are shown in Fig. 2a, b, respectively. Aluminum alloy ingot of $1.25 \mathrm{~kg}$ charged into the electric furnace and
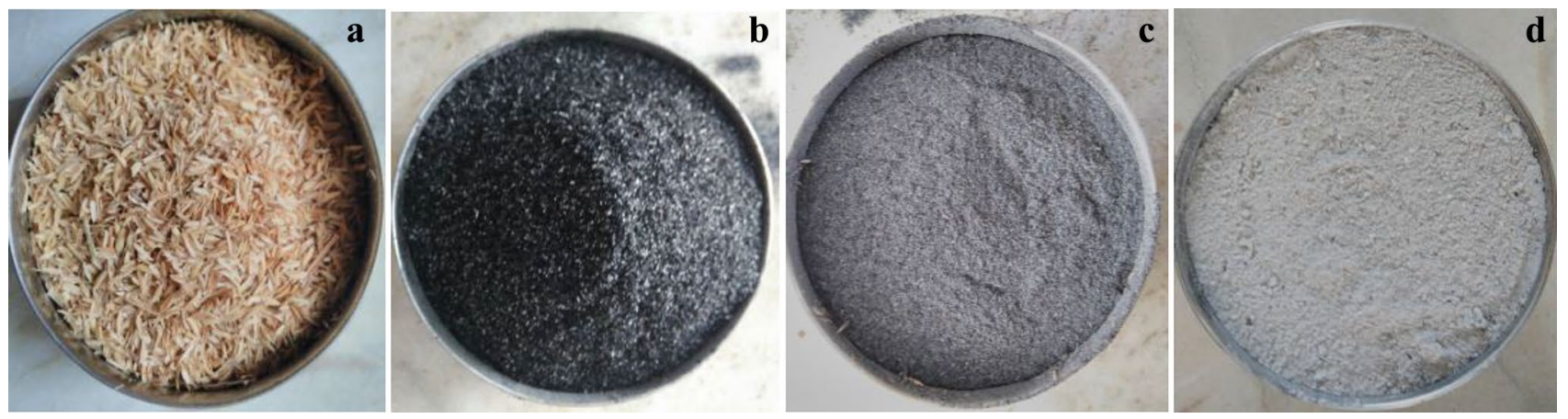

Fig. 1 Different stages of RHA. a Rice husk, b carbonized RHA, c sieve RHA, d heat treated RHA

\section{SN Applied Sciences}



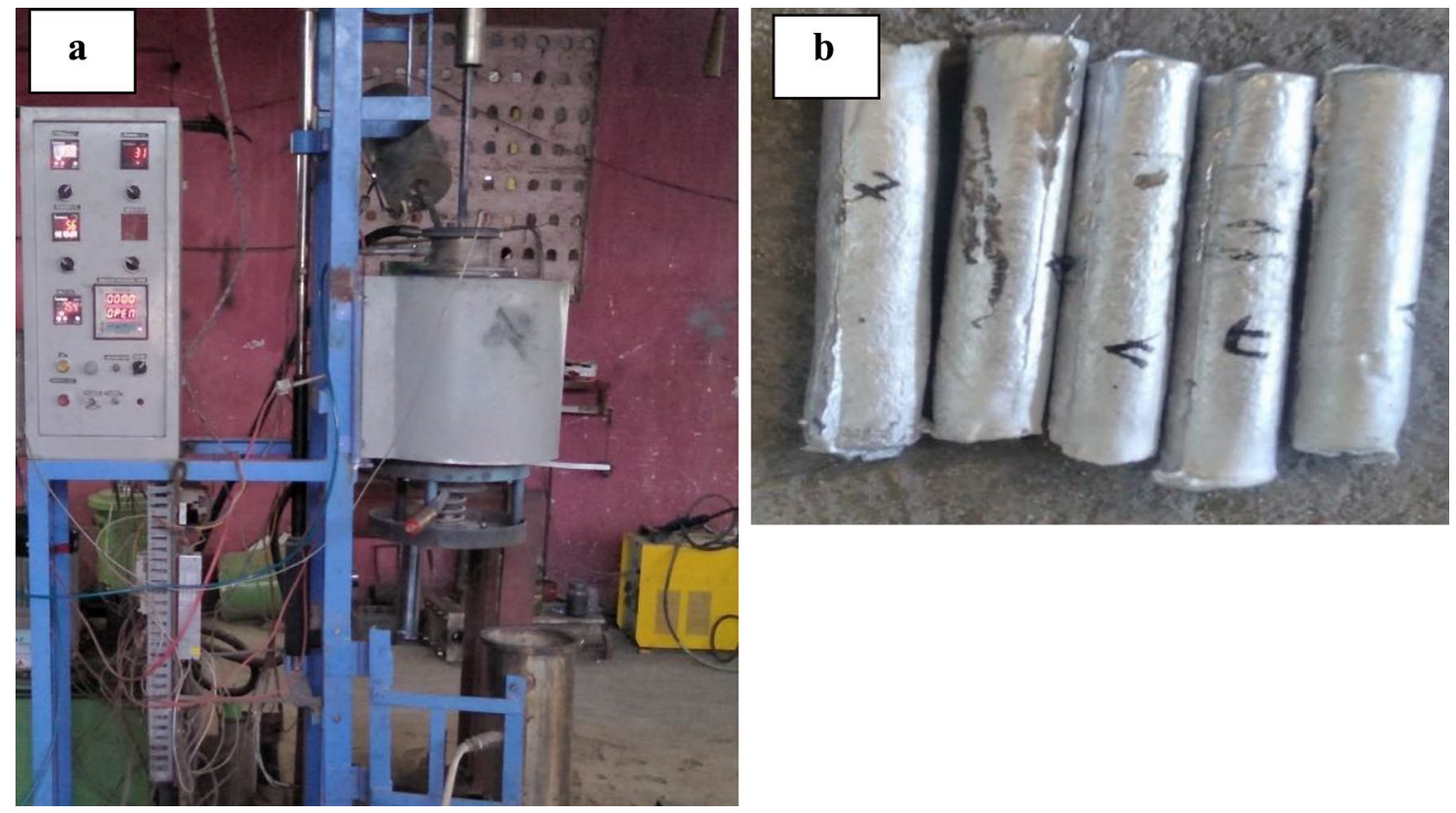

Fig. 2 a Model of stir casting equipment, $\mathbf{b}$ fabricated PHAMCs

is heated to its melting temperature. Reinforcement particulates are preheated to a temperature range of $300^{\circ} \mathrm{C}$ to remove moisture and dampness. Magnesium ribbon ( $6 \mathrm{~mm}$ length) is added to the molten metal to improve wettability between matrix and reinforcement particulates. In the first stage, preheated TiC particles are added slowly to the molten metal under the stirring condition of $300 \mathrm{rpm}$. The mixture is continuously stirred for $2 \mathrm{~min}$. In the second stage preheated RHA particles are added slowly to the molten vortex and the molten mixture is stirred at a speed of $300 \mathrm{rpm}$ for $5 \mathrm{~min}$. To avoid shrinkage and to get uniform solidification, gray cast iron mould is preheated to $450{ }^{\circ} \mathrm{C}$. Weight percentages $(3 \%+3 \%$, $5 \%+5 \%, 7 \%+7 \%$, and $9 \%+9 \%$ ) of TiC and RHA are equally changed to get composite at various proportions. Finally the fabricated hybrid composite of 3\%, 5\%, $7 \%$ and $9 \%$ weight ratio for each composition are named as $\mathrm{S} 1, \mathrm{~S} 2, \mathrm{~S} 3$, and $\mathrm{S} 4$, respectively. $\mathrm{T} 6$ temper is done by homogenizing the material at $400{ }^{\circ} \mathrm{C}$ for $1 \mathrm{~h}$, and the aged at $115^{\circ} \mathrm{C}$ for $24 \mathrm{~h}$. The matrix and its composition are given in Table 1.
The specific gravity of prepared PHAMCs are measured using Mikro-tech instruments. The Mitutoyo HM-200B made Micro-Vickers hardness tester is used to measure the hardness values. The Electronic Tensiometer test rig (Kudale instrument) of ASTM: E08 is used for tensile test. Charpy V-notch test is used to measure the toughness of PHAMCs.

\section{Results and discussion}

\subsection{X-ray diffraction and microstructural characterization}

XRD and EDS techniques are used to measure the silicon content in RHA and to verify the presence of other foreign elements in the composition. $\mathrm{RHA}$ having $\mathrm{SiO}_{2}$ content in the range of $94-96 \%$ is successfully extracted and it is shown in Fig. 3a. XRD pattern of composite samples i.e. S1, S2, S3, S4 are shown in Fig. 3b. Principle elements like Al,

Table 1 Details of PHAMCs

\begin{tabular}{|c|c|c|c|c|c|c|c|c|c|c|}
\hline Code & Composition & & & & & & & & & \\
\hline \multirow[t]{2}{*}{ Nasent Sample (S0) } & Elements & Si & $\mathrm{Fe}$ & $\mathrm{Cu}$ & $\mathrm{Mn}$ & $\mathrm{Mg}$ & $\mathrm{Ch}$ & $\mathrm{Zn}$ & $\mathrm{Ti}$ & Al 6061 \\
\hline & Composition \% & 0.4 & 0.7 & 0.02 & 0.07 & 1.1 & 0.3 & 0.15 & 0.09 & Rest \\
\hline Sample 1 (S1) & \multicolumn{10}{|c|}{ AA6061 mix with 3\% RHA and 3\% TiC } \\
\hline Sample 2 (S2) & \multicolumn{10}{|c|}{ AA6061 mix with $5 \%$ RHA and $5 \% \mathrm{TiC}$} \\
\hline Sample 3 (S3) & \multicolumn{10}{|c|}{ AA6061 mix with 7\% RHA and 7\% TiC } \\
\hline Sample 4 (S4) & \multicolumn{10}{|c|}{ AA6061 mix with 9\% RHA and 9\% TiC } \\
\hline
\end{tabular}




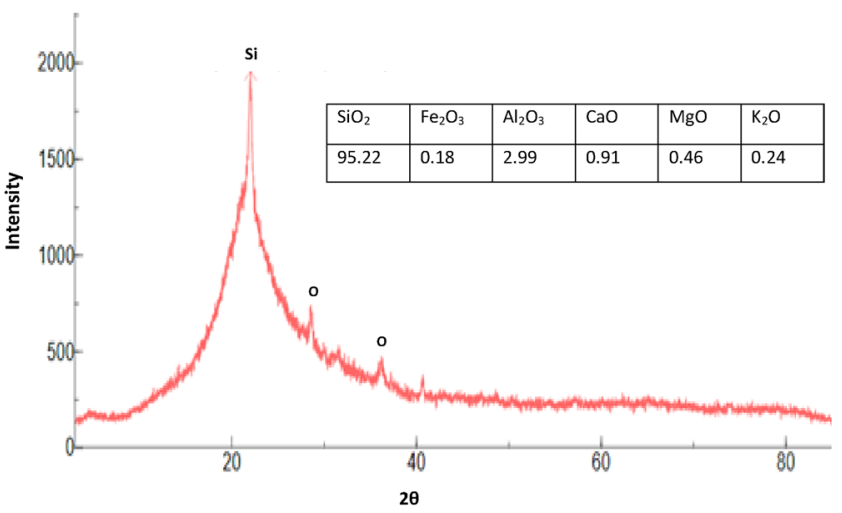

a XRD pattern of RHA

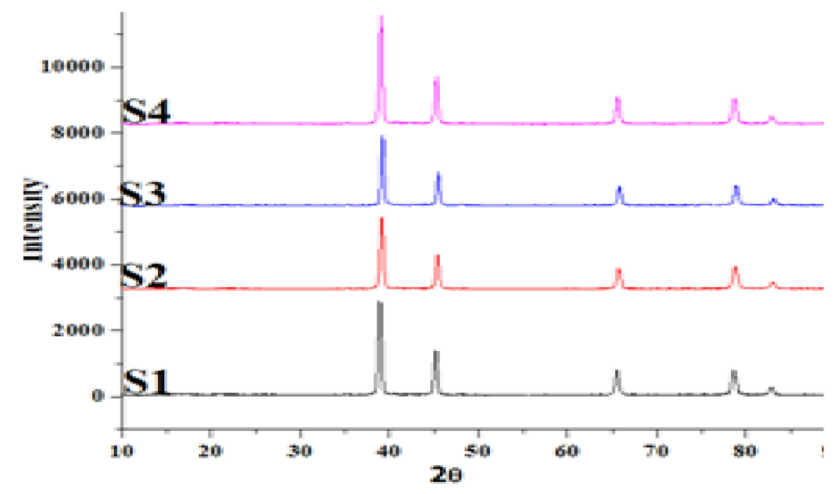

b XRD pattern of Developed PHAMCs

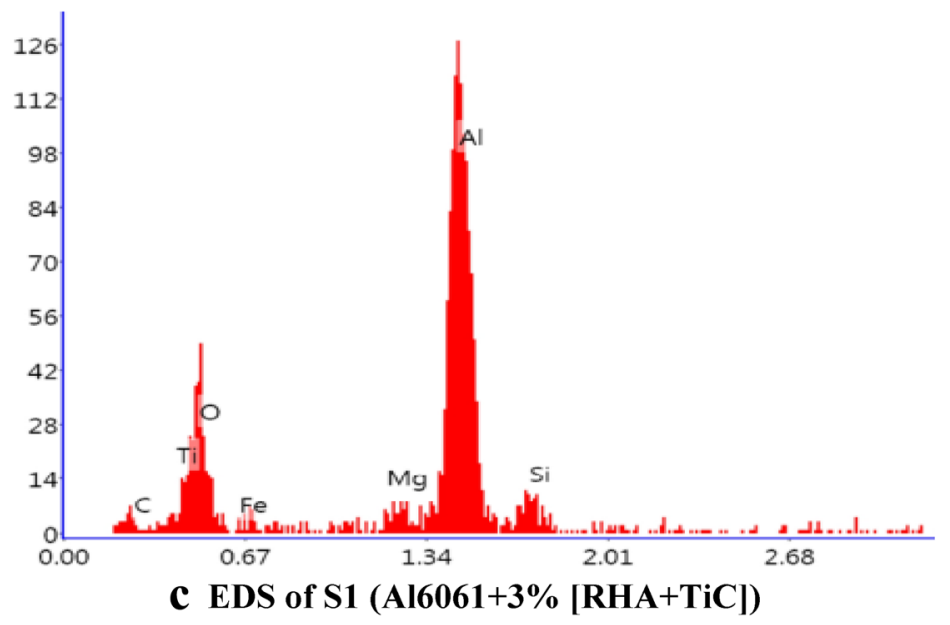

Fig. 3 XRD of RHA and EDS of composite

$\mathrm{TiC}$ and $\mathrm{SiO}_{2}$ are present in PHAMCs without any intermetallic elements. Also, the presence of TiC and RHA elements in the hybrid composite are shown in Fig. 3c. The peaks of $\mathrm{SiO}_{2}$ and $\mathrm{TiC}$ increased with the reinforcement material weight fractions.

To understand the particle distributions in PHAMC samples are subjected to optical microscopic examination. The microstructure image of S1, S2, S3 and S4 are shown in Fig. 4a-d. RHA particles are distributed along the grain boundary. As RHA is said to be pore filling elements, it moves to the grain boundary and fills the space between the bonding elements [18]. The uniform distribution of particles without agglomerations are found in S1 and S2. Increase in RHA content in the matrix leads to have agglomerations and this can be verified from Fig. 4c, d of S3 and S4. Due to agglomeration, materials in that region possess poor strength and porosity. The presence of small quantity of Magnesium in Al 6061 alloy and addition of magnesium content in the matrix improve the wettability of the composite. Excellent interfacial bonding between matrix alloy and reinforcement particles is achieved with the addition of Mg. The distribution of TiC particle and RHA along the grain boundary are indicated in Fig. 4e.

\subsection{Measurement of density and estimated porosity}

Using Archimedes principle density of PHAMCs with different weight fractions of $\mathrm{RHA}+\mathrm{TiC}$ are calculated. $\mathrm{S} 3$ sample shows a decrease density of $3.88 \%$. Fabricate composite exhibits low densities in addition of RHA particles as reinforcements. Theoretical and experimental analysis on density reveals that with increase in RHA leads to high porosity. XRD observation reveals that RHA contains $95 \%$ of $\mathrm{SiO}_{2}$, when added as reinforcement results in porosity and reduced hardness due to the presence of excess oxygen content in RHA. The effect of reinforcement particles on density is shown in Fig. 5 . Estimated percentage of porosity and density for various weight ratios of the fabricated hybrid composites are shown in Table 2. 

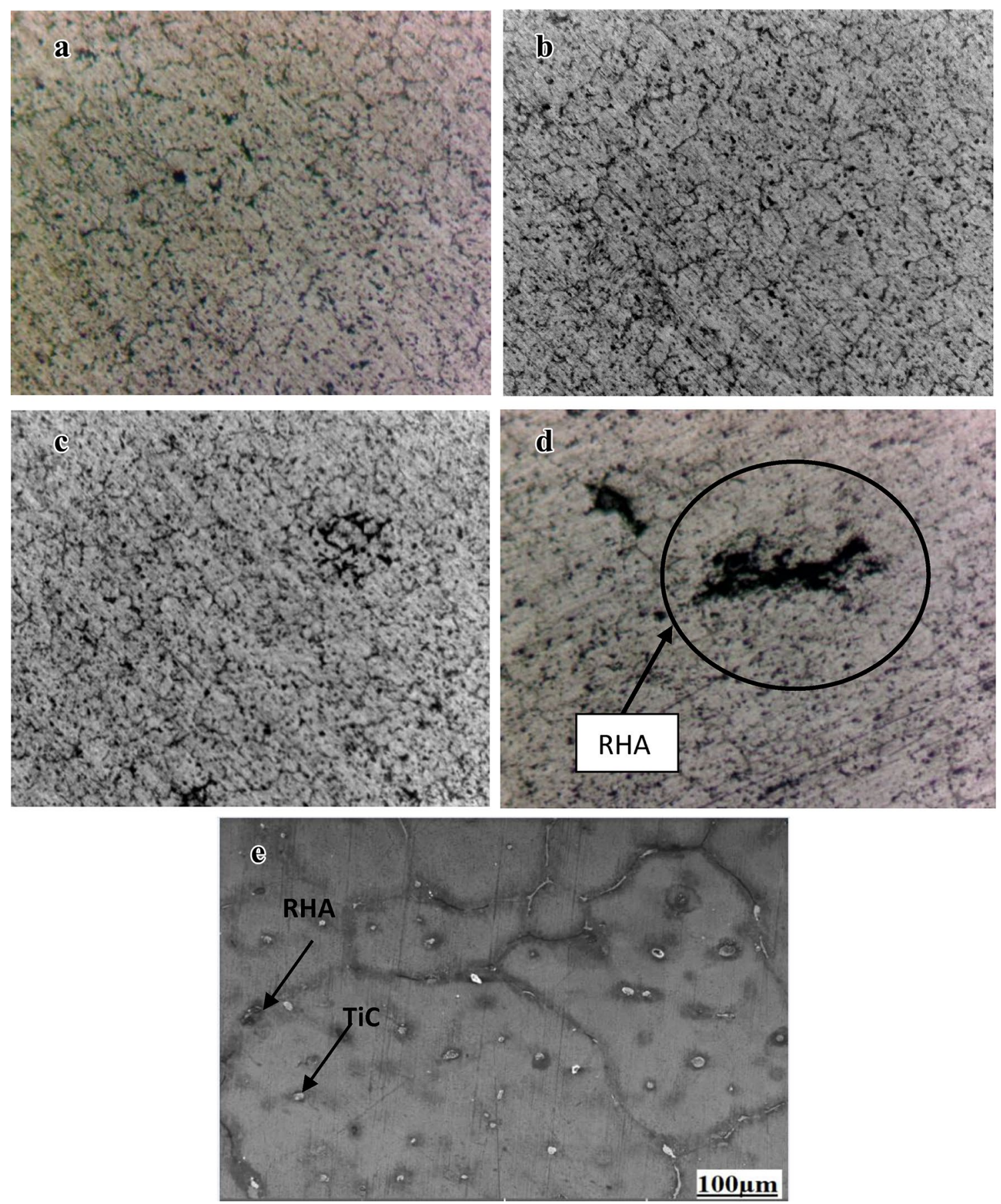

Fig. 4 Optical microscopy of fabricated samples. a S1, b S2, c S3, d S4 and e micrograph of Al $6061+7$ wt\% of TiC and RHA

\subsection{Measurement of micro-Vickers hardness}

Hardness of the samples are tested as per ASTM-E 384 standards. Samples are disc polished and etched using reagent Keller's $(1.0 \mathrm{ml} \mathrm{HF}, 1.5 \mathrm{ml} \mathrm{HCl}, 2.5 \mathrm{ml} \mathrm{HNO}, 95 \mathrm{ml}$ water) by swabbing $15 \mathrm{~s}$. A load of $500 \mathrm{~g}$ for a dwell time of $15 \mathrm{~s}$ is applied on samples at ambient temperature. Ten indentations are made at different locations on each sample then the average values are recorded as hardness for the particular sample. From the observation, it can be stated that the hardness observation increases up to 7\% weight fractions (S3 sample) because of high composite strain energy. The hardness increases due to grain reinforcement, thermal expansion coefficient between matrix alloy and reinforcement particles i.e. at interfacial's strains mismatch. Micro-hardness of the 


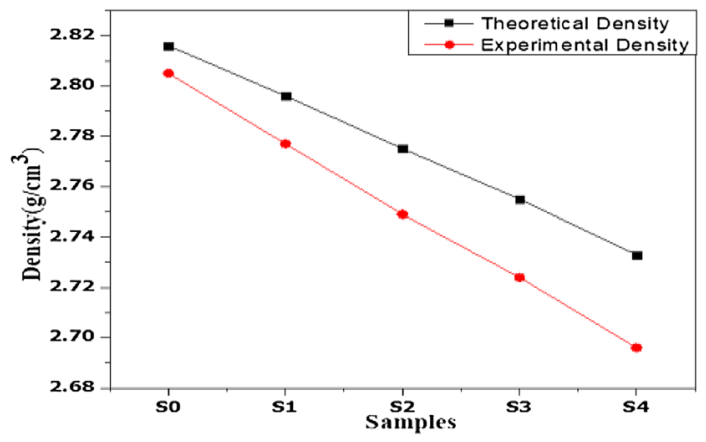

Fig. 5 The density of developed PHAMCs

Table 2 Estimated percentage porosity of fabricated hybrid composites

\begin{tabular}{llll}
\hline Sample & $\begin{array}{l}\text { Theoretical den- } \\
\text { sity }\left(\mathrm{g} / \mathrm{cc}^{3}\right)\end{array}$ & $\begin{array}{l}\text { Experimental den- } \\
\text { sity }\left(\mathrm{g} / \mathrm{cc}^{3}\right)\end{array}$ & $\begin{array}{l}\text { Percentage } \\
\text { porosity }\end{array}$ \\
\hline S0 & 2.816 & 2.805 & 0.3906 \\
S1 & 2.796 & 2.777 & 0.6795 \\
S2 & 2.775 & 2.749 & 0.9369 \\
S3 & 2.755 & 2.724 & 1.1252 \\
S4 & 2.733 & 2.696 & 1.3538 \\
\hline
\end{tabular}

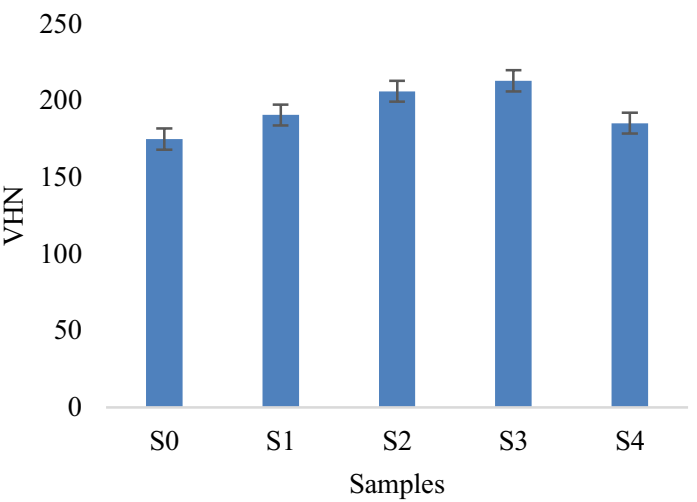

Fig. 6 Variations of Vickers hardness values of the PHAMC
PHAMCs decreases on the further increase of reinforcement particles because of agglomerations and porosity. The observation of micro Vickers hardness results is shown in Fig. 6.

\subsection{Tensile test}

Tensile strength of the test samples increases up to S3 sample due to fine grains arrangement, minimal shear load effect, arrested dislocation movements, particle strengthening, and interfacial strains mismatch. Ceramic particles improves stress transformations from the matrix to the reinforcement particle because of the Orowan mechanism [3]. The interactions between the dislocations and reinforcement particles has improved the tensile strength of the composite. The tensile strength decreases on further addition of reinforcement particles which is mainly due to agglomerations and porous that lead to grain detachments at low load condition. The increases of hard ceramic and refractory particles in the matrix lead to have low percentage of elongation and create the plastic deformation surface. Observation of tensile test and the percentage of elongation is shown in Fig. 7a, b, respectively.

\subsection{Impact test}

To determine the energy absorption rate of PHAMCs, the samples are subjected to Charpy impact test as per ASTME23. The effect of reinforcement on aluminum hybrid composite for the energy absorption rate is shown in Fig. 8 . Increase in reinforcement weight fractions will decrease the ductility of samples and increases the stress concentrations which will create micro-cracks within the elemental composition. From the Charpy test, it could be confirmed that Impact strength of samples decreases with addition of reinforcement in the matrix. Excess amount of reinforcements progressively increases the hardness, reduction in ductile property, micro-cracks within the grain and in grain boundary which are due to stress concentration.
Fig. 7 a Variations of tensile strength, $\mathbf{b}$ change in percentage of elongation

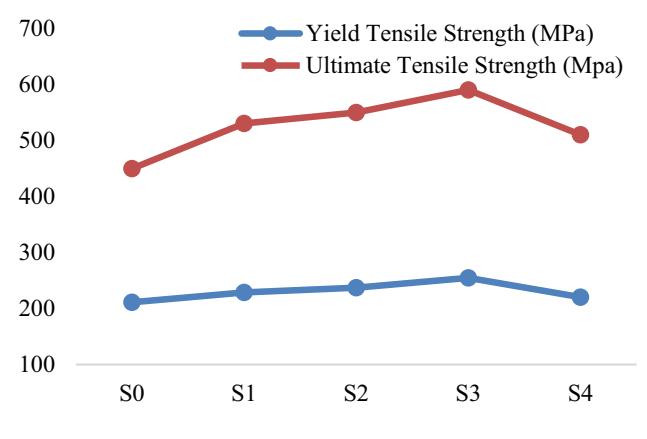

$\mathbf{a}$

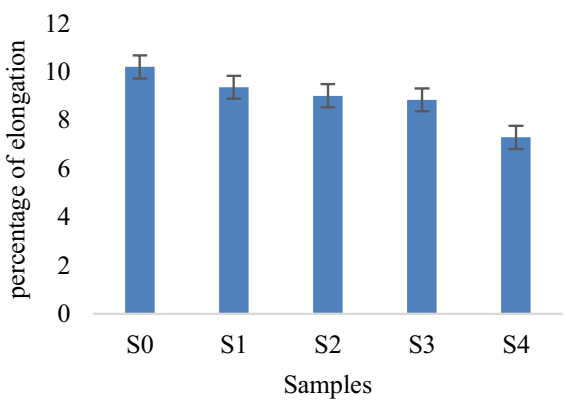

b 


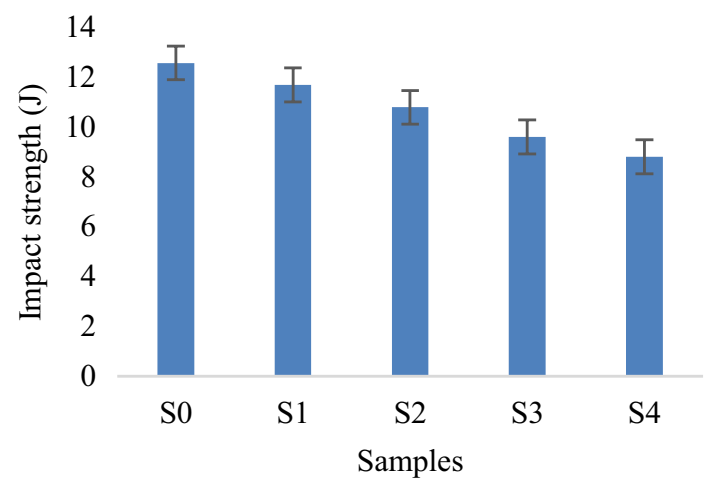

Fig. 8 Variations of impact strength values of PHAMCs

\subsection{Fractography}

The fracture surfaces of the impact test samples are analyzed using scanned electron microscopy. SEM analysis revealed that RHA particles have an internal skeleton structure of silica and necklace-shaped cross-section with aluminum dendrites. There is no continuity in the dendritic structure across RHA particles. The SEM images for tempered condition samples are shown at higher and lower magnifications in Fig. 9a-h. Addition of 3\% RHA-TiC are shown in Fig. 9a, b indicates failure due to dimples and delamination. $5 \%$ of reinforcement samples shown in Fig. 9c, d clearly indicates the failure mechanism is mainly due to the cluster of particles, voids with minor cracks that leads to have plastic deformation surface. From the morphology of the fractured section which is shown in Fig. 9e, fimplies that increase of reinforcements will have mixed mode of failure with transgranular cleavage facets, reinforcement fracture, and dimples. Change from ductile to brittle mode is observed on increasing in percentage of reinforcement particles Fig. 9g, h.

\section{Conclusion}

Aluminum matrix with different weight percentage of TiC and RHA are successfully prepared through stir casting process. TiC and RHA improve the mechanical properties of the PHAMCs compared to the nascent sample at tempered condition. Some important observations from the above experimental study are as follows:

- Addition of reinforcement's decreases the percentage of elongation and impact strength to a maximum of $28.52 \%$ and $29.91 \%$ respectively.

- Developed PHAMCs with 7\% weight fractions tends to have decreased density by $3.88 \%$ compared to unreinforced aluminum 6061.

- Ultimate tensile strength, yield strength, and Micro Vickers hardness of PHAMCs increased to $31 \%, 17.1 \%$, and $18 \%$ respectively with addition of $7 \mathrm{wt} \%$ reinforcement.

- Beyond $7 \mathrm{wt} \%$ reinforcement, the mechanical properties such as tensile strength, impact energy, hardness, and density of the PHAMCs decreases.

- Microstructural analysis on PHAMCs shows agglomerations and increase in porosity with RHA. Mixed mode of failure, reinforcement fracture, dimples, micro-cracks are found on the fractured surface. 

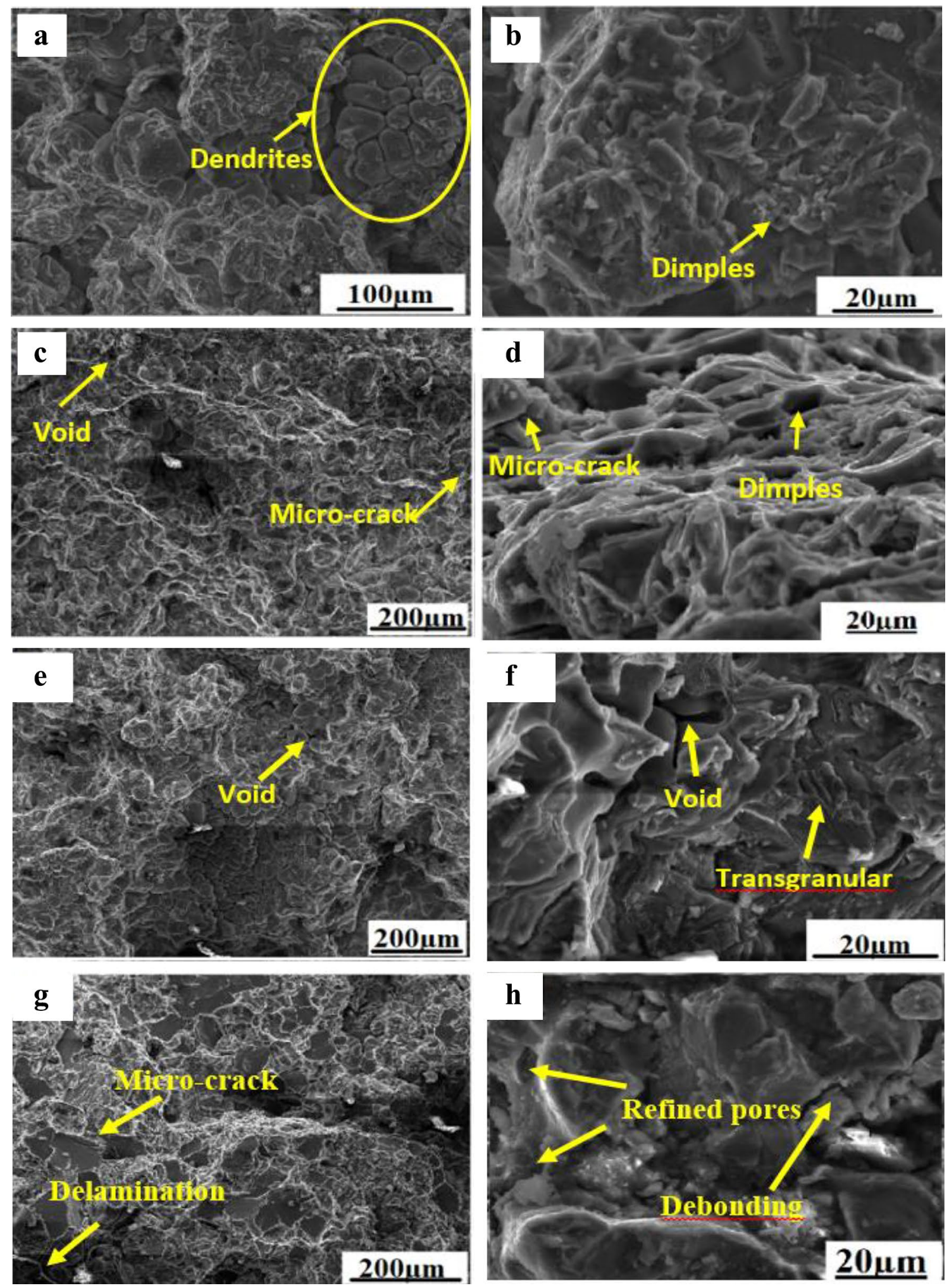

Fig. 9 a-h Impact fracture samples with various weight fractions of reinforcements; $\mathbf{a}, \mathbf{b} 3$ wt\% RHA and TiC, $\mathbf{c}, \mathbf{d} 5$ wt\% RHA and TiC, e, $\mathbf{f}$ 7 wt $\%$ RHA and TiC, g, h 9 wt\% RHA and TiC 
Acknowledgements The authors are grateful to the Centre of Excellence at VFSTR (Deemed to be University) for rendering their support and guidance to finish this work.

\section{Compliance with ethical standards}

Conflict of interest The authors declare that they have no conflict of interest.

\section{References}

1. Nicholls CJ, Boswell B, Davies IJ, Islam MN (2017) Review of machining metal matrix composites. Int J Adv Manuf Technol 90:2429-2441

2. Rebba $B$, Ramanaiah N (2015) Investigations on mechanical behaviour of $\mathrm{B}_{4} \mathrm{C}$ and $\mathrm{MoS}_{2}$ reinforced $\mathrm{AA} 2024$ hybrid composites. J Manuf Sci Prod 15:339-343

3. Mohan A, Narendra K, Rakesh K, Mohan S (2017) High-temperature tribology of AA5052/ZrB ${ }_{2}$ PAMCs. JTribol 139(1):011601-12

4. Subramaniam B, Natarajan B, Kaliyaperumal B, Jerold S (2018) Investigation on mechanical properties of aluminium 6061boron carbide-coconut shell fly ash reinforced hybrid metal matrix composites. China Found 15(6):449-456

5. Kumar M, Murugan AM (2019) Tribological characterization of Al 6061/alumina/graphite/redmud hybrid composite for brake rotor application. Part Sci Technol 37(3):261-274

6. Arora G (2018) A comparative study of AA6351 mono-composites reinforced with synthetic and agro waste reinforcement. Int J Precis Eng Manuf 19(4):631-638

7. Arora $\mathrm{G}$ (2017) A review on monolithic and hybrid metal-matrix composites reinforced with industrial-agro wastes. J Braz Soc Mech Sci Eng 39(11):4819-4835

8. Unlu BS (2008) Investigation of tribological and mechanical properties $\mathrm{Al}_{2} \mathrm{O}_{3}-\mathrm{SiC}$ reinforced $\mathrm{Al}$ composites manufactured by casting or P/M method. Mater Des 29(10):2002-2008
9. Suthar J, Patel KM (2018) Processing issues, machining and applications of aluminum metal matrix composites. Mater Manuf Process 33(5):499-527

10. Raju SS, Panigrahi MK, Ganguly RI, Rao GS (2019) Tribological behaviour of Al-1100-coconut shell ash (CSA) composite at elevated temperature. Tribol Int 129:55-66

11. Verma N, Vettivel SC (2018) Characterization and experimental analysis of boron carbide and rice husk ash reinforced AA6061 aluminium alloy hybrid composite. J Alloys Compd 741:981-998

12. Ravikumar K, Kiran K, Sreebalaji VS (2017) Micro structural characteristics and mechanical behaviour of aluminium matrix composite reinforced with titanium carbide. J Alloys Compd 723:795-801

13. Shoba C, Prasad DS, Prathipati R (2019) Investigations on the machinability of $\mathrm{Al} / \mathrm{SiC} / \mathrm{RHA}$ hybrid metal matrix composites. Silicon 11(47):1-12

14. Prasad DS (2012) Production and mechanical properties of A356.2/RHA composites. J Mater Sci Technol 28(4):367-372

15. Veeravalli RR, Ramanaiah N, Sarcar MM (2016) Mechanical and tribology properties of AA7075-TiC metal matrix composites under heat treated (T6) and cast conditions. J Mater Res Technol 5(4):377-383

16. Hossain MR, Ali MH, Amin MA, Kibria MG, Ferdous MS (2017) Fabrication and performance test of aluminium alloy-rice husk ash hybrid metal matrix composite as industrial and construction material. Int J Eng Mater Manuf 2:94-102

17. Prasad DS, Shoba C, Ramanaiah N (2014) Investigations on mechanical properties of aluminum hybrid composites. J Mater Res Technol 3:79-85

18. Rodrigues CS, Ghavami K, Stroeven P (2010) Rice Husk Ash as a supplementary raw material for the production of cellulosecement composites with improved performance. J Mater Sci 41:6925-6937

Publisher's Note Springer Nature remains neutral with regard to jurisdictional claims in published maps and institutional affiliations. 